\title{
CryoEM Structure of Dynamin-like MxB in Assembly
}

Peijun Zhang ${ }^{1,2,3,4}$, Frances Joan D. Alvarez ${ }^{3,4}$, Shaoda He ${ }^{5}$, Juan R. Perilla ${ }^{6}$, Sooin Jang ${ }^{4,7,8}$, Alan N. Engelman ${ }^{4,7,8}$, Sjors H.W. Scheres ${ }^{5}$

${ }^{1}$ Division of Structural Biology, University of Oxford, The Henry Wellcome Building for Genomic Medicine, Headington, Oxford OX3 7BN, UK

2Electron Bio-Imaging Centre, Diamond Light Sources, Harwell Science and Innovation Campus, Didcot OX11 ODE, UK

${ }^{3}$ Department of Structural Biology, ${ }^{4}$ Pittsburgh Center for HIV Protein Interactions, University of Pittsburgh School of Medicine, Pittsburgh, PA 15260, USA

${ }^{5}$ MRC Laboratory of Molecular Biology, Francis Crick Avenue, Cambridge CB2 0QH, UK

${ }^{6}$ Department of Physics and Beckman Institute, University of Illinois at Urbana-Champaign, Urbana, IL 61801, USA

${ }^{7}$ Department of Cancer Immunology and Virology, Dana-Farber Cancer Institute, Boston, MA 02215, USA

${ }^{8}$ Department of Medicine, Harvard Medical School, Boston, MA 02215, USA

Human dynamin-like, interferon-induced myxovirus resistance $2(\mathrm{Mx} 2 / \mathrm{MxB})$ is a potent inhibitor of HIV-1 infection and a potential agent for the treatment of HIV/AIDS. MxB directly interacts with the HIV-1 capsid and blocks nuclear import of pre-integration complexes and subsequent chromosomal integration of the viral cDNA. Anti-HIV-1 activity and capsid-binding require the $\mathrm{N}$-terminal domain of $\mathrm{MxB}$ and protein oligomerization, yet each of these has eluded structural determination due to difficulties in protein preparation. We purified a GTPase active MBP fusion of the full-length wild-type MxB from mammalian cells. The full-length MxB purifies as discrete oligomers and further self-assembles into helical arrays in physiological salt. Intriguingly, GTP, but not GDP, binding to $\mathrm{MxB}$ results in array disassembly, while subsequent GTP hydrolysis allows its re-formation. Using cryoEM, we determined the MxB assembly structure at $4.6 \AA$ resolution, revealing novel oligomerization and higher-order assembly interfaces that were absent or are distinct from the crystal structure of $\mathrm{MxB}$ that lacked the Nterminal domain and harbored interface mutations. The structure suggests that salt bridges mediate MxB higher-order assembly, which is disrupted by GTP-induced conformational changes. More importantly, mutational analysis combined with viral infectivity assays revealed that $\mathrm{MxB}$ oligomers, not the dimer or higher-order assemblies, are in fact the active species against HIV-1 infection. The near-atomic wild-type MxB structure provides a vital framework for re-evaluating conflicting results regarding the effects of $\mathrm{MxB}$ oligomerization and GTPase activity on its anti-HIV-1 activity. Moreover, this first high-resolution assembly structure among the superfamily of dynamin-like large GTPases allows us to propose a new GTP-dependent assembly/disassembly model, distinct from current models. 